1

2

3

\title{
The Shapley value for directed graph games
}

\author{
Anna Khmelnitskaya* $\quad$ Özer Selçuk $^{\dagger} \quad$ Dolf Talman $^{\ddagger}$
}

December 8, 2015

\begin{abstract}
The Shapley value for directed graph (digraph) TU games with limited cooperation induced by a digraph prescribing the dominance relation among the players is introduced. It is defined as the average of the marginal contribution vectors corresponding to all permutations which do not violate the induced subordination of players. We study properties of this solution and its core stability. For digraph games with the digraphs being directed cycles an axiomatization of the solution is obtained.
\end{abstract}

Keywords: TU game; Shapley value; directed graph; dominance structure; core; convexity

JEL Classification Number: C71

\section{Introduction}

In classical cooperative game theory it is assumed that any coalition of players may form and is able to obtain payoffs for its members. Problem is how much payoff each player should receive. However, in many practical situations the set of feasible coalitions is limited by some social, economical, hierarchical, or technical structure. One of the most famous singleton solutions for cooperative games with transferable utility (TU games), where payoffs can be distributed freely among the players, is the Shapley value [9] defined as the average of the marginal contribution vectors corresponding to all permutations on the players. Several adaptations of the Shapley value for models of games with limited cooperation among the players are well known in the literature, cf. Aumann and Drèze [1] and Owen [8] for games with coalition structure, Myerson [7] for games with cooperation structure introduced by means of undirected graphs in which only the connected players are able to cooperate. For games with limited cooperation that is described in terms of (cycle-free) directed graphs (digraphs) we mention Gilles and Owen [4] for games with permission structure using the disjunctive approach and Gilles at al. [5] for such games using the conjunctive approach, and Faigle and Kern [2] for games with precedence constraints.

In this paper we assume that restricted cooperation is determined by an arbitrary digraph on the player set, the directed links of which prescribe the subordination among the players. For example, consider a society consisting of individuals with different opinions, possibly incomplete preferences, about the importance of several proposals or tasks that

\footnotetext{
*A.B. Khmelnitskaya, Saint-Petersburg State University, Faculty of Applied Mathematics, e-mail: a.b.khmelnitskaya@utwente.nl

${ }^{\dagger}$ Ö. Selçuk, University of Portsmouth, Portsmouth Business School, e-mail: ozer.selcuk@port.ac.uk

$\ddagger_{\text {}}$ A.J.J. Talman, Tilburg University, CentER, Department of Econometrics \& Operations Research, email: talman@tilburguniversity.edu
} 
need to be completed. If the preferences of the individuals are aggregated by using majority voting, then it is well known that the resulting structure will be a directed graph on the set of alternatives. In this directed graph, a directed link from one proposal to another proposal means that the majority of the society thinks that the former one is more important than the latter one. If it is assumed that at each moment only one proposal or task can be performed, then when one is completed, the next one to be performed can be any of its immediate successors in the digraph or one of those the performance of which does not depend on it. In this example the digraph might not be cycle-free because directed cycles may stand for the well known Condorcet paradox.

On the class of digraph games, which are games with restricted cooperation determined by a digraph prescribing the dominance relation on the set of players, we introduce the so-called Shapley value for digraph games as the average of marginal contribution vectors corresponding to all permutations not violating the subordination of players. Contrary to the Myerson model, the feasible coalitions are not necessarily connected. We show that the Shapley value for digraph games meets efficiency, linearity, the restricted null player property, the restricted equal treatment property, is independent of inessential links, and is stable with respect to the appropriate core concept under a convexity type condition which is weaker than the usual convexity guaranteeing the core stability of the classical Shapley value. On the subclass of cycle digraph games for which the digraphs are directed cycles an axiomatization is provided.

Since precedence constraints are determined by a partial ordering on the player set which can be represented by a cycle-free digraph, the games under precedence constraints form a subclass of cycle-free digraph games on which the Shapley value for digraph games coincides with the Shapley value for games under precedence constraints of Faigle and Kern [2]. There is no straightforward relation of permission values for games with permission structure with the newly introduced Shapley value for digraph games. In games with permission structure players need permission from their predecessors in order to cooperate, at least one of them for disjunctive approach and all of them for conjunctive approach. In both cases a permission-restricted TU game is derived from the given TU game taking into account the permission structure and the disjunctive and conjunctive permission values for games with permission structure are defined as the Shapley value of the corresponding permission-restricted games.

The structure of the paper is as follows. Section 2 contains preliminaries. Section 3 introduces the Shapley value for digraph games and discusses its properties and stability. An axiomatization on the subclass of cycle digraph games is obtained in Section 4.

\section{Preliminaries}

A cooperative game with transferable utility (TU game) is a pair $(N, v)$, where $N=\{1, \ldots, n\}$ is a finite set of $n \geq 2$ players and $v: 2^{N} \rightarrow \mathbb{R}$ is a characteristic function with $v(\emptyset)=0$, assigning to any coalition $S \subseteq N$ its worth $v(S)$. The set of TU games with fixed player set $N$ is denoted $\mathcal{G}_{N}$. For simplicity of notation and if no ambiguity appears we write $v$ when we refer to a game $(N, v)$. It is well known (cf. Shapley [9]) that unanimity games $\left\{u_{T}\right\}_{\substack{T \subseteq N \\ T \neq \emptyset}}$, defined as $u_{T}(S)=1$ if $T \subseteq S$, and $u_{T}(S)=0$ otherwise, form a basis in $\mathcal{G}_{N}$. A value on $\mathcal{G} \subseteq \mathcal{G}_{N}$ is a function $\xi: \mathcal{G} \rightarrow \mathbb{R}^{N}$ that assigns to every $v \in \mathcal{G}$ a vector $\xi(v) \in \mathbb{R}^{N}$ where $\xi_{i}(v)$ is the payoff to $i \in N$ in $v$. The marginal contribution of $i \in N$ to $S \subseteq N \backslash\{i\}$ in $v \in \mathcal{G}_{N}$ is given by $m_{i}^{v}(S)=v(S \cup\{i\})-v(S)$. In the sequel we use standard notation 

$x(S)=\sum_{i \in S} x_{i}$ for any $x \in \mathbb{R}^{N}$ and $S \subseteq N$.

For a permutation $\pi: N \rightarrow N, \pi(i)$ is the position of player $i \in N$ in $\pi, P_{\pi}(i)=\{j \in$ $N \mid \pi(j)<\pi(i)\}$ is the set of predecessors of $i$ in $\pi$, and $\bar{P}_{\pi}(i)=P_{\pi}(i) \cup\{i\}$. In what follows we identify a permutation $\pi$ with the vector $(\pi(1), \ldots, \pi(n))$. Let $\Pi$ be the set of permutations on $N$. For $v \in \mathcal{G}_{N}$ and $\pi \in \Pi$ the marginal contribution vector $\bar{m}^{v}(\pi) \in \mathbb{R}^{N}$ is given by $\bar{m}_{i}^{v}(\pi)=m_{i}^{v}\left(P_{\pi}(i)\right)=v\left(\bar{P}_{\pi}(i)\right)-v\left(P_{\pi}(i)\right)$ for all $i \in N$. The Shapley value of $v \in \mathcal{G}^{N}$ is given by $S h(v)=\sum_{\pi \in \Pi} \bar{m}^{v}(\pi) / n$ !.

A graph on $N$ consists of $N$ as the set of nodes and for a directed graph (digraph) a collection of ordered pairs $\Gamma \subseteq\{(i, j) \mid i, j \in N, i \neq j\}$ as the set of directed links (arcs) from one player to another in $N$, and for an undirected graph a collection of unordered pairs $\Gamma \subseteq\{\{i, j\} \mid i, j \in N, i \neq j\}$ as the set of links (edges) between two players in $N$. Observe that an undirected graph can be considered as a digraph for which $(i, j) \in \Gamma$ iff $(j, i) \in \Gamma$. We say that a digraph $\Gamma$ contains an undirected link $\{i, j\}$ and write $\{i, j\} \in \Gamma$ if $(i, j),(j, i) \in \Gamma$. The set of digraphs on fixed $N$ we denote $\Gamma_{N}$. For $\Gamma \in \Gamma_{N}$ and $S \subseteq N$, $\left.\Gamma\right|_{S}=\{(i, j) \in \Gamma \mid i, j \in S\}$ is the subgraph of $\Gamma$ on $S$. Given $\Gamma \in \Gamma_{N}$ a sequence of different players $\left(i_{1}, \ldots, i_{r}\right), r \geq 2$, is a path in $\Gamma$ between $i_{1}$ and $i_{r}$ if $\left\{\left(i_{h}, i_{h+1}\right),\left(i_{h+1}, i_{h}\right)\right\} \cap \Gamma \neq \emptyset$ for $h=1, \ldots, r-1$, and a directed path in $\Gamma$ from $i_{1}$ to $i_{r}$ if $\left(i_{h}, i_{h+1}\right) \in \Gamma$ for $h=1, \ldots, r-1$. A directed path $\left(i_{1}, \ldots, i_{r}\right)$ is a directed cycle if $\left(i_{r}, i_{1}\right) \in \Gamma$ and when $r \geq 3$, both the path does not contain undirected links and $\left(i_{1}, i_{r}\right) \notin \Gamma$. $\Gamma$ is cycle-free if it contains no directed cycles. Players $i, j \in N$ are connected in $\Gamma$ if there exists a path in $\Gamma$ between $i$ and $j$. $\Gamma$ is connected if any $i, j \in N, i \neq j$, are connected in $\Gamma . S \subseteq N$ is connected in $\Gamma$ if $\left.\Gamma\right|_{S}$ is connected. For $S \subseteq N, C^{\Gamma}(S)$ denotes the collection of subsets of $S$ connected in $\Gamma, S / \Gamma$ is the collection of maximal connected subsets, called components, of $S$ in $\Gamma$. For $i, j \in N$ if there exists a directed path in $\Gamma$ from $i$ to $j$, then $j$ is a successor of $i$ and $i$ is a predecessor of $j$ in $\Gamma$. If $(i, j) \in \Gamma$, then $j$ is an immediate successor of $i$ and $i$ is an immediate predecessor of $j$ in $\Gamma$. For $i \in N, S^{\Gamma}(i)$ denotes the set of successors of $i$ in $\Gamma$ and $\bar{S}^{\Gamma}(i)=S^{\Gamma}(i) \cup\{i\}$. A chain on $N$ is a connected cycle-free digraph on $N$ in which each player has at most one immediate successor and one immediate predecessor.

For $\Gamma \in \Gamma_{N}, S \subseteq N$ and $i, j \in S, i$ dominates $j$ in $\left.\Gamma\right|_{S}$, denoted $i \succ_{\left.\Gamma\right|_{S}} j$, if $j \in S^{\left.\Gamma\right|_{S}}(i)$ and $i \notin S^{\left.\Gamma\right|_{S}}(j)$. Observe that the dominance relation between two players may differ between different coalitions they both belong to. Player $i \in S$ is undominated in $\left.\Gamma\right|_{S}$ if no player in $S$ dominates $i$ in $\left.\Gamma\right|_{S}$, i.e., $i \in S^{\left.\Gamma\right|_{S}}(j)$ implies $j \in S^{\left.\Gamma\right|_{S}}(i)$. Note that a player undominated in $\left.\Gamma\right|_{S}$ either has no predecessor in $\left.\Gamma\right|_{S}$ or lies on a directed cycle in $\left.\Gamma\right|_{S} \cdot U^{\Gamma}(S)$ denotes the set of players undominated in $\left.\Gamma\right|_{S}$. Since $N$ is finite, $U^{\Gamma}(S) \neq \emptyset$ for $\emptyset \neq S \subseteq N$.

A pair $(v, \Gamma)$ of $v \in \mathcal{G}_{N}$ and $\Gamma \in \Gamma_{N}$ constitutes a directed graph game, or a digraph game. The set of digraph games on fixed $N$ is denoted $\mathcal{G}_{N}^{\Gamma}$. A value on $\mathcal{G} \subseteq \mathcal{G}_{N}^{\Gamma}$ is a function $\xi: \mathcal{G} \rightarrow \mathbb{R}^{N}$ assigning to every $(v, \Gamma) \in \mathcal{G}$ a payoff vector $\xi(v, \Gamma)$.

\section{The Shapley value for digraph games}

In a digraph game the digraph prescribes a dominance relation between the players that puts restrictions on the feasibility of coalitions. Assuming that in order to cooperate players may join only the players not dominating them, the set of feasible coalitions of a digraph game consists of hierarchical coalitions.

Given $\Gamma \in \Gamma_{N}, S \subseteq N$ is a hierarchical coalition in $\Gamma$ if $i \in S,(i, j) \in \Gamma$, and $i \notin S^{\Gamma}(j)$ imply $\bar{S}^{\Gamma}(j) \subset S$. 
If a player in a hierarchical coalition dominates an immediate successor, then the coalition also contains this latter player and all his successors. Every hierarchical coalition preserves the subordination of players and therefore is feasible. For a cycle-free $\Gamma \in \Gamma_{N}$, $S \subseteq N$ is hierarchical iff every successor of any $i \in S$ in $\Gamma$ belongs to $S$, i.e., $\bar{S}^{\Gamma}(i) \subseteq S$ for all $i \in S$. So, for a cycle-free digraph the set of hierarchical coalitions coincides with the set of feasible coalitions in Faigle and Kern [2] when the precedence constraints are induced by the same digraph. Note that both the empty and grand coalitions are hierarchical. A hierarchical coalition is not necessarily connected. In an undirected graph, in particular in the empty graph, every coalition is hierarchical. For $\Gamma \in \Gamma_{N}, H(\Gamma)$ denotes the set of coalitions hierarchical in $\Gamma$ and $H^{c}(\Gamma)$ its subset of all connected coalitions. Observe that $S, T \in H(\Gamma)$ implies $S \cup T, S \cap T \in H(\Gamma)$.

Given $\Gamma \in \Gamma_{N}, \pi \in \Pi$ is consistent with $\Gamma$ if it preserves the subordination of players determined by $\Gamma$, i.e., $\pi(j)<\pi(i)$ only if $j \nsucc_{\Gamma_{\bar{P}_{\pi}(i)}} i$.

For $\Gamma \in \Gamma_{N}, \Pi^{\Gamma}$ denotes the set of permutations consistent with $\Gamma$. Since $N$ is finite, $\Pi^{\Gamma} \neq \emptyset$.

Remark 3.1 For every $\pi \in \Pi^{\Gamma}$ each player is undominated in the subgraph of $\Gamma$ on the set composed by this player and his predecessors in $\pi$, i.e., $i \in U^{\Gamma}\left(\bar{P}_{\pi}(i)\right)$ for all $i \in N$.

The next proposition shows that every consistent permutation generates a sequence of feasible coalitions consisting of a player and his predecessors in the permutation.

Proposition 3.1 Given $\Gamma \in \Gamma_{N}$, if $\pi \in \Pi^{\Gamma}$, then $\bar{P}_{\pi}(i), P_{\pi}(i) \in H(\Gamma)$ for all $i \in N$.

Proof. First note that (i) $N \in H(\Gamma)$, (ii) $N=\bar{P}_{\pi}(h)$ for some $h \in N$, and (iii) for each $i \in N$ it holds that $P_{\pi}(i)=\bar{P}_{\pi}(j)$ for $j \in P_{\pi}(i)$ such that $\pi(j)=\max _{k \in P_{\pi}(i)} \pi(k)$. So, it suffices to show that if $\bar{P}_{\pi}(k) \in H(\Gamma)$ for some $k \in N$, then $P_{\pi}(k) \in H(\Gamma)$ as well. If $\bar{P}_{\pi}(k) \in H(\Gamma)$, then $i \in P_{\pi}(k),(i, j) \in \Gamma$, and $i \notin S^{\Gamma}(j)$ imply $\bar{S}^{\Gamma}(j) \subset \bar{P}_{\pi}(k)$. To prove that $P_{\pi}(k) \in H(\Gamma)$ we show $k \notin \bar{S}^{\Gamma}(j)$. Suppose $k \in \bar{S}^{\Gamma}(j)$. Then $(i, j) \in \Gamma$ implies $k \in S^{\Gamma}(i)$;

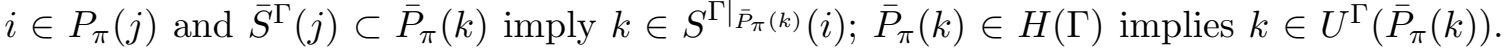

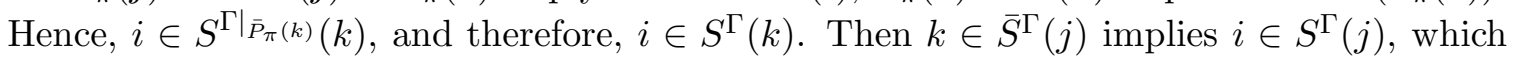
contradicts $i \notin S^{\Gamma}(j)$.

Remark 3.2 If $\Gamma \in \Gamma_{N}$ is a directed cycle, then for all $\pi \in \Pi^{\Gamma}$ and $i \in N$ both $\bar{P}_{\pi}(i)$ and $P_{\pi}(i)$ are connected in $\Gamma$. Moreover, $U^{\Gamma}(N)=N$ and $U^{\Gamma}\left(\bar{P}_{\pi}(i)\right)=\{i\}$ if $\bar{P}_{\pi}(i) \neq N$.

We define the Shapley value for digraph games as the average of the marginal contribution vectors corresponding to all consistent permutations, i.e., for any $(v, \Gamma) \in \mathcal{G}_{N}^{\Gamma}$,

$$
S h(v, \Gamma)=\frac{1}{\left|\Pi^{\Gamma}\right|} \sum_{\pi \in \Pi^{\Gamma}} \bar{m}^{v}(\pi) .
$$

Example 3.1 Consider the 5-player digraph games $(v, \Gamma),\left(v, \Gamma^{\prime}\right)$, and $\left(v, \Gamma^{\prime \prime}\right)$ with characteristic function $v(S)=|S|^{2}$ for all $S \subseteq N$ and digraphs as depicted in Figure 1.

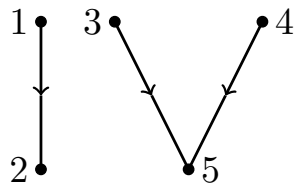

a) digraph $\Gamma$

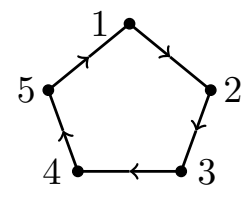

b) digraph $\Gamma^{\prime}$

Figure 1

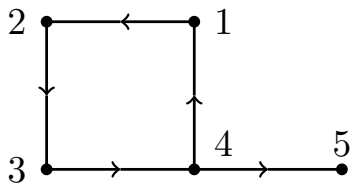

c) digraph $\Gamma^{\prime \prime}$ 
There are 20 permutations consistent with $\Gamma: \pi^{1}=(5,4,3,2,1), \pi^{2}=(5,3,4,2,1), \pi^{3}=$ $(5,4,2,3,1), \pi^{4}=(5,2,4,3,1), \pi^{5}=(2,5,4,3,1), \pi^{6}=(5,3,2,4,1), \pi^{7}=(5,2,3,4,1), \pi^{8}=$ $(2,5,3,4,1), \pi^{9}=(5,2,4,1,3), \pi^{10}=(2,5,4,1,3), \pi^{11}=(5,4,2,1,3), \pi^{12}=(5,2,1,4,3), \pi^{13}=$ $(2,5,1,4,3), \pi^{14}=(2,1,5,4,3), \pi^{15}=(5,2,3,1,4), \pi^{16}=(2,5,3,1,4), \pi^{17}=(5,3,2,1,4)$, $\pi^{18}=(5,2,1,3,4), \pi^{19}=(2,5,1,3,4), \pi^{20}=(2,1,5,3,4)$, and $\operatorname{Sh}(v, \Gamma)=(7,3,13 / 2,13 / 2,2)$. There are 5 permutations consistent with $\Gamma^{\prime}: \pi^{1}=(5,4,3,2,1), \pi^{2}=(4,3,2,1,5), \pi^{3}=$ $(3,2,1,5,4), \pi^{4}=(2,1,5,4,3), \pi^{5}=(1,5,4,3,2)$, and $\operatorname{Sh}\left(v, \Gamma^{\prime}\right)=(5,5,5,5,5)$. There are 10 permutations consistent with $\Gamma^{\prime \prime}: \pi^{1}=(5,4,3,2,1), \pi^{2}=(5,1,4,3,2), \pi^{3}=(5,3,2,1,4)$, $\pi^{4}=(5,2,1,4,3), \pi^{5}=(1,5,4,3,2), \pi^{6}=(2,1,5,4,3), \pi^{7}=(2,5,1,4,3), \pi^{8}=(3,2,1,5,4)$, $\pi^{9}=(3,5,2,1,4), \pi^{10}=(3,2,5,1,4)$, and $S h\left(v, \Gamma^{\prime \prime}\right)=(5,2,4.6,5.2,7,3)$. To compare, the Shapley value of $v$ is the average of 120 marginal contribution vectors determined by all $\pi \in \Pi$ and $S h(v)=(5,5,5,5,5)$. Due to the symmetry of both $v$ and $\Gamma^{\prime}, S h\left(v, \Gamma^{\prime}\right)=S h(v)$.

When $\Gamma \in \Gamma_{N}$ represents an undirected graph, i.e., there is no subordination between the players in $\Gamma$, the Shapley value of $(v, \Gamma) \in \mathcal{G}_{N}^{\Gamma}$ coincides with the Shapley value of $v$. Both values also coincide if $v$ is symmetric and $\Gamma$ is a directed cycle, as for $\left(v, \Gamma^{\prime}\right)$ in Example 3.1. In general, the Shapley value of a digraph game does not coincide with the Myerson value [7] of the corresponding undirected graph game because the Myerson value is defined as the average of all marginal contribution vectors of the Myerson restricted game. Since a cycle-free digraph on the player set provides a partial ordering of the players and for a cycle-free digraph the set of hierarchical coalitions coincides with the set of feasible coalitions in Faigle and Kern [2], on the subclass of cycle-free digraph games the Shapley value for digraph games coincides with the Shapley value for cooperative games under precedence constraints defined in Faigle and Kern [2]. Moreover, if for a connected digraph game all covering trees of the digraph are chains, the Shapley value for digraph games coincides with the average covering tree value introduced in Khmelnitskaya, Selcuk, and Talman [6]. In particular, this holds for cycle digraph games for which the digraph is a directed cycle.

A value $\xi$ on $\mathcal{G} \subseteq \mathcal{G}_{N}^{\Gamma}$ is efficient $(E)$ if for any $(v, \Gamma) \in \mathcal{G}, \sum_{i \in N} \xi_{i}(v, \Gamma)=v(N)$.

A value $\xi$ on $\mathcal{G} \subseteq \mathcal{G}_{N}^{\Gamma}$ is linear $(L)$ if for any $(v, \Gamma),(w, \Gamma) \in \mathcal{G}$ and $a, b \in \mathbb{R}, \xi(a v+$ $b w, \Gamma)=a \xi(v, \Gamma)+b \xi(w, \Gamma)$, where $(a v+b w)(S)=a v(S)+b w(S)$ for all $S \subseteq N$.

A value $\xi$ on $\mathcal{G} \subseteq \mathcal{G}_{N}^{\Gamma}$ satisfies the restricted equal treatment property $(R E T P)$ if for any $(v, \Gamma) \in \mathcal{G}$ and $i, j \in N, i \neq j$, hierarchically symmetric in $(v, \Gamma)$ it holds that $\xi_{i}(v, \Gamma)=\xi_{j}(v, \Gamma)$.

Players $i, j \in N, i \neq j$, are hierarchically symmetric in $(v, \Gamma) \in \mathcal{G}_{N}^{\Gamma}$ if they are both symmetric in $\Gamma$ and hierarchically symmetric in $v$. Players $i, j \in N, i \neq j$, are symmetric in $\Gamma$ if they have the same sets of immediate successors and immediate predecessors in $\Gamma$, i.e., $(i, k) \in \Gamma \Longleftrightarrow(j, k) \in \Gamma$ and $(k, i) \in \Gamma \Longleftrightarrow(k, j) \in \Gamma$. Players $i, j \in N, i \neq j$, are hierarchically symmetric in $v$ if for all $S \subseteq N \backslash\{i, j\}$ such that $S, S \cup\{i\}, S \cup\{j\}, S \cup\{i, j\} \in$ $H(\Gamma)$, it holds that $v(S \cup\{i\})=v(S \cup\{j\})$, or, equivalently, $m_{i}^{v}(S)=m_{j}^{v}(S)$.

A value $\xi$ on $\mathcal{G} \subseteq \mathcal{G}_{N}^{\Gamma}$ meets the (restricted) hierarchical null-player property $((R) H N P)$ if for all $(v, \Gamma) \in \mathcal{G}, \xi_{i}(v, \Gamma)=0$ whenever $i$ is a (restricted) hierarchical null-player in $(v, \Gamma)$.

A player $i \in N$ is a (restricted) hierarchical null-player in $(v, \Gamma) \in \mathcal{G}_{N}^{\Gamma}$ if for every $S \subseteq N \backslash\{i\}$ such that $S, S \cup\{i\} \in H(\Gamma)\left(S, S \cup\{i\} \in H^{c}(\Gamma)\right)$, it holds that $v(S \cup\{i\})=v(S)$, or, equivalently, $m_{i}^{v}(S)=0$.

Remark 3.3 Each null-player in $v \in \mathcal{G}_{N}$ is a hierarchical null-player in any $(v, \Gamma) \in \mathcal{G}_{N}^{\Gamma}$, and every hierarchical null-player in $(v, \Gamma) \in \mathcal{G}_{N}^{\Gamma}$ is also a restricted hierarchical null-player in $(v, \Gamma)$, i.e., RHNP implies HNP. 
A value $\xi$ on $\mathcal{G} \subseteq \mathcal{G}_{N}^{\Gamma}$ is (restricted) hierarchically marginalist $((R) H M)$ if for any $(v, \Gamma),(w, \Gamma) \in \mathcal{G}$ and $i \in N$ for which $m_{i}^{v}(S)=m_{i}^{w}(S)$ for all $S \subseteq N \backslash\{i\}$ such that $S, S \cup\{i\} \in H(\Gamma)\left(S, S \cup\{i\} \in H^{c}(\Gamma)\right)$ and $i \in U^{\Gamma}(S \cup\{i\}), \xi_{i}(v, \Gamma)=\xi_{i}(w, \Gamma)$.

If in a cycle digraph game only the grand coalition is productive, then due to symmetry of the players on the cycle it is natural to require that they all get the same payoff.

A value $\xi$ on $\mathcal{G} \subseteq \mathcal{G}_{N}^{\Gamma}$ is strongly symmetric on directed cycles $(S S D C)$ if for any $(v, \Gamma) \in \mathcal{G}$ such that $\Gamma$ is a directed cycle on $N$ and $v(S)=0$ for all $S \subsetneq N$, i.e., $v=\lambda u_{N}$ for some real $\lambda, \xi_{i}(v, \Gamma)=\xi_{j}(v, \Gamma)$ for all $i, j \in N, i \neq j$.

A value $\xi$ on $\mathcal{G} \subseteq \mathcal{G}_{N}^{\Gamma}$ is independent of inessential directed links (IIDL) if for any $(v, \Gamma) \in \mathcal{G}$ and inessential directed link $(i, j) \in \Gamma, \xi(v, \Gamma)=\xi(v, \Gamma \backslash\{(i, j)\})$.

For $\Gamma \in \Gamma_{N},(i, j) \in \Gamma$ is inessential if $i$ dominates $j$ in $\Gamma$ and there exists a directed path in $\Gamma$ from $i$ to $j$ different from $(i, j)$, i.e., $i \notin S^{\Gamma}(j)$ and there exists $i^{\prime} \in N$ such that $\left(i, i^{\prime}\right) \in \Gamma, i \notin S^{\Gamma}\left(i^{\prime}\right)$, and $j \in S^{\Gamma}\left(i^{\prime}\right)$.

Proposition 3.2 The Shapley value for digraph games on $\mathcal{G}_{N}^{\Gamma}$ meets $E, L, R E T P, H N P$, $H M, S S D C$, and IIL.

Proof. (E) This follows from the efficiency of all marginal contribution vectors on $\mathcal{G}_{N}$.

(L) Since $(v, \Gamma),(w, \Gamma)$ and $(a v+b w, \Gamma)$ are determined by the same $\Gamma, \Pi^{\Gamma}$ is the same for all. Then $\mathrm{L}$ follows from the linearity of all marginal contribution vectors on $\mathcal{G}_{N}$.

(RETP) Let $i, j \in N$ be hierarchically symmetric in $(v, \Gamma) \in \mathcal{G}_{N}^{\Gamma}$. Then $\pi \in \Pi^{\Gamma}$ iff $\pi^{\prime} \in \Pi^{\Gamma}$, where $\pi^{\prime}(i)=\pi(j), \pi^{\prime}(j)=\pi(i)$, and $\pi^{\prime}(k)=\pi(k)$ for all $k \in N \backslash\{i, j\}$. So, it suffices to show that $\bar{m}_{i}^{v}(\pi)=\bar{m}_{j}^{v}\left(\pi^{\prime}\right)$ and $\bar{m}_{j}^{v}(\pi)=\bar{m}_{i}^{v}\left(\pi^{\prime}\right)$ for any such $\pi$ and $\pi^{\prime}$. Without loss of generality assume that $\pi(i)>\pi(j)$. To show $\bar{m}_{i}^{v}(\pi)=\bar{m}_{j}^{v}\left(\pi^{\prime}\right)$ note that $\pi^{\prime}(i)=\pi(j)$ and $\pi^{\prime}(k)=\pi(k)$ for all $k \in N \backslash\{i, j\}$ imply $\bar{P}_{\pi}(i)=\bar{P}_{\pi^{\prime}}(j)$ and $P_{\pi}(i) \backslash\{j\}=P_{\pi^{\prime}}(j) \backslash\{i\}$. Let $S=P_{\pi}(i) \backslash\{j\}$. By Proposition 3.1, $S \cup\{i\}, S \cup\{j\}, S \cup\{i, j\} \in H(\Gamma)$. Since $i$ and $j$ are hierarchically symmetric in $v, v(S \cup\{i\})=v(S \cup\{j\})$, i.e., $v\left(P_{\pi}(i)\right)=v\left(P_{\pi^{\prime}}(j)\right)$. This together with $\bar{P}_{\pi}(i)=\bar{P}_{\pi^{\prime}}(j)$ implies $\bar{m}_{i}^{v}(\pi)=v\left(\bar{P}_{\pi}(i)\right)-v\left(P_{\pi}(i)\right)=v\left(\bar{P}_{\pi^{\prime}}(j)\right)-v\left(P_{\pi^{\prime}}(j)\right)=$ $\bar{m}_{j}^{v}\left(\pi^{\prime}\right)$. To show $\bar{m}_{j}^{v}(\pi)=\bar{m}_{i}^{v}\left(\pi^{\prime}\right)$ observe that $P_{\pi}(j)=P_{\pi^{\prime}}(i)$. Let $S=P_{\pi}(j)$. By Proposition 3.1, $S \cup\{i\}, S \cup\{j\}, S \in H(\Gamma)$. Since $i$ and $j$ are hierarchically symmetric in $v, v(S \cup\{i\})=v(S \cup\{j\})$, i.e., $v\left(\bar{P}_{\pi}(j)\right)=v\left(\bar{P}_{\pi^{\prime}}(i)\right)$. So, $\bar{m}_{j}^{v}(\pi)=v\left(\bar{P}_{\pi}(j)\right)-v\left(P_{\pi}(j)\right)=$ $v\left(\bar{P}_{\pi^{\prime}}(i)\right)-v\left(P_{\pi^{\prime}}(i)\right)=\bar{m}_{i}^{v}\left(\pi^{\prime}\right)$.

(HNP) Let $i \in N$ be a hierarchical null player in $(v, \Gamma) \in \mathcal{G}_{N}^{\Gamma}$ and $\pi \in \Pi^{\Gamma}$. By Proposition 3.1, $\bar{P}_{\pi}(i), P_{\pi}(i) \in H(\Gamma)$. Then, $\bar{m}_{i}^{v}(\pi)=v\left(\bar{P}_{\pi}(i)\right)-v\left(P_{\pi}(i)\right)=0$. Hence, $S h_{i}(v, \Gamma)=0$.

(HM) This follows from (1), Remark 3.1, and Proposition 3.1.

(SSDC) This holds true because game $\lambda u_{N}$ is symmetric, $S h_{i}\left(\lambda u_{N}\right)=\lambda / n$ for all $i \in N$, and for any cycle digraph game $(v, \Gamma)$ with symmetric $v, S h(v, \Gamma)=S h(v)$.

(IIDL) Let $(v, \Gamma) \in \mathcal{G}_{N}^{\Gamma}$ for which $(i, j) \in \Gamma$ is inessential. Then there exists $i^{\prime} \in N$ such that $\left(i, i^{\prime}\right) \in \Gamma, i \notin S^{\Gamma}\left(i^{\prime}\right)$, and $j \in S^{\Gamma}\left(i^{\prime}\right)$. Let $\Gamma^{\prime}=\Gamma \backslash\{(i, j)\}$. We show now that $\Pi^{\Gamma}=\Pi^{\Gamma^{\prime}}$, so that $S h(v, \Gamma)=S h\left(v, \Gamma^{\prime}\right)$. Take $\pi \in \Pi^{\Gamma}$ and suppose $\pi \notin \Pi^{\Gamma^{\prime}}$. Since $\pi \notin \Pi^{\Gamma^{\prime}}$, there exist $k, k^{\prime} \in N, \pi\left(k^{\prime}\right)<\pi(k)$, such that $k^{\prime} \succ_{\left.\Gamma^{\prime}\right|_{P_{\pi}(k)}} k$, i.e., $k \in S^{\left.\Gamma^{\prime}\right|_{P_{\pi}(k)}\left(k^{\prime}\right)}$

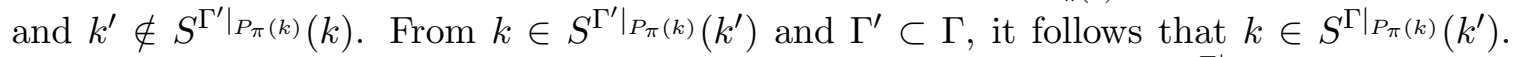
By Remark 3.1, $k \in U^{\Gamma}\left(\bar{P}_{\pi}(k)\right)$ because $\pi \in \Pi^{\Gamma}$, and therefore, $k^{\prime} \in S^{\left.\Gamma\right|_{P_{\pi}(k)}(k) \text {. Whence }}$ it follows that in $\left.\Gamma\right|_{P_{\pi}(k)}$ there is a path from node $k$ to node $k^{\prime}$. Both $\left.k^{\prime} \notin S^{\Gamma^{\prime}}\right|_{P_{\pi}(k)}(k)$

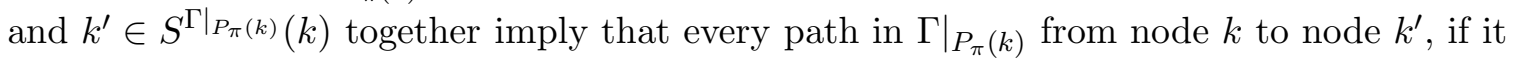
exists, should contain link $(i, j)$. But due to Proposition 3.1, $P_{\pi}(k) \in H(\Gamma)$, and therefore, for each path in $\left.\Gamma\right|_{P_{\pi}(k)}$ from $k$ to $k^{\prime}$ containing $(i, j)$ there exists another path in $\left.\Gamma\right|_{P_{\pi}(k)}$ from $k$ to $k^{\prime}$, in which link $(i, j)$ is replaced by the path from $i$ to $j$ via node $i^{\prime}$, which 
leads to a contradiction. So, $\pi \in \Pi^{\Gamma}$ implies $\pi \in \Pi^{\Gamma^{\prime}}$. Take now $\pi^{\prime} \in \Pi^{\Gamma^{\prime}}$ and suppose $\pi^{\prime} \notin \Pi^{\Gamma}$. Since $\pi^{\prime} \notin \Pi^{\Gamma}$, there exist $k, k^{\prime} \in N, \pi^{\prime}\left(k^{\prime}\right)<\pi^{\prime}(k)$, such that $k^{\prime} \succ_{\Gamma_{P_{\pi^{\prime}}(k)}} k$, i.e., $k \in S^{\left.\Gamma\right|_{P_{\pi^{\prime}}(k)}}\left(k^{\prime}\right)$ and $k^{\prime} \notin S^{\left.\Gamma\right|_{\pi_{\pi^{\prime}}}(k)}(k)$. From $k^{\prime} \notin S^{\left.\Gamma\right|_{P_{\pi^{\prime}}(k)}}(k)$ and $\Gamma^{\prime} \subset \Gamma$, it follows

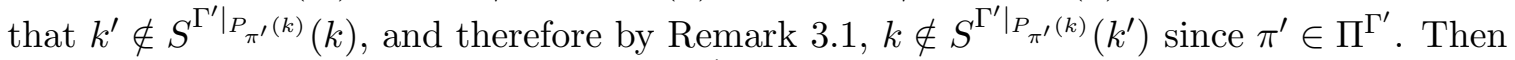
the conditions $k \in S^{\left.\Gamma\right|_{\pi_{\pi^{\prime}}(k)}}\left(k^{\prime}\right)$ and $k \notin S^{\left.\Gamma^{\prime}\right|_{P_{\pi^{\prime}}(k)}}\left(k^{\prime}\right)$ together, similarly as above, lead to a contradiction, which proves that $\pi^{\prime} \in \Pi^{\Gamma^{\prime}}$ implies $\pi^{\prime} \in \Pi^{\Gamma}$.

Under the assumption that in a digraph game the digraph represents the dominance structure on the player set, only the hierarchical coalitions are feasible. So, we define the dominance core $C^{D}(v, \Gamma)$ of $(v, \Gamma) \in \mathcal{G}_{N}^{\Gamma}$ as the set of efficient payoff vectors that cannot be blocked by any hierarchical coalition, i.e., $C^{D}(v, \Gamma)=\left\{x \in \mathbb{R}^{N} \mid x(N)=v(N), x(S) \geq\right.$ $v(S)$ for all $S \in H(\Gamma)\}$.

A value $\xi$ on $\mathcal{G} \subseteq \mathcal{G}_{N}^{\Gamma}$ is $D$-stable if for every $(v, \Gamma) \in \mathcal{G}, \xi(v, \Gamma) \in C^{D}(v, \Gamma)$.

A digraph game $(v, \Gamma) \in \mathcal{G}_{N}^{\Gamma}$ is hierarchically convex if for any $S, T \in H(\Gamma), v(S)+v(T) \leq$ $v(S \cup T)+v(S \cap T)$.

Remark that the hierarchical convexity for $(v, \Gamma)$ is weaker than convexity for $v$ where the inequality is required to hold for all $S, T \subseteq N$.

Theorem 3.1 The Shapley value for digraph games is D-stable on the class of hierarchically convex digraph games.

Proof. Let $(v, \Gamma) \in \mathcal{G}_{N}^{\Gamma}$ be a hierarchically convex digraph game. Since the Shapley value for digraph games is efficient, it suffices to show that $\sum_{i \in S} \bar{m}_{i}^{v}(\pi) \geq v(S)$ for every $S \in H(\Gamma)$ and $\pi \in \Pi^{\Gamma}$. Take any $S \in H(\Gamma)$ and $\pi \in \Pi^{\Gamma}$, and let $S_{1}, \ldots, S_{k}$ partition $S$ such that $S_{h}=\left\{i \in S \mid \pi\left(b_{h}\right) \leq \pi(i) \leq \pi\left(a_{h}\right)\right\}, h=1, \ldots, k$, where the numbers $a_{h}$ and $b_{h}, h=1, \ldots, k$, satisfy $\pi\left(a_{h-1}\right)+1<\pi\left(b_{h}\right) \leq \pi\left(a_{h}\right)$, with $\pi\left(a_{0}\right)=-1$. Define $\bar{P}_{\pi}\left(a_{0}\right)=\emptyset$. For any $h \in\{1, \ldots, k\}$ consider the sets $S \cup \bar{P}_{\pi}\left(a_{h-1}\right)$ and $P_{\pi}\left(b_{h}\right)$. By Proposition 3.1 and since $S$ is hierarchical, both sets are hierarchical coalitions. Moreover, their intersection is equal to $\bar{P}_{\pi}\left(a_{h-1}\right)$ and their union is equal to $S \cup \bar{P}_{\pi}\left(a_{h}\right)$. Hierarchical convexity implies

$$
v\left(S \cup \bar{P}_{\pi}\left(a_{h}\right)\right)+v\left(\bar{P}_{\pi}\left(a_{h-1}\right)\right) \geq v\left(S \cup \bar{P}_{\pi}\left(a_{h-1}\right)\right)+v\left(P_{\pi}\left(b_{h}\right)\right) .
$$

By repeated application of this inequality for $h=1, \ldots k$, we obtain

$$
v\left(S \cup \bar{P}_{\pi}\left(a_{k}\right)\right)+\sum_{h=1}^{k} v\left(\bar{P}_{\pi}\left(a_{h-1}\right)\right) \geq v\left(S \cup \bar{P}_{\pi}\left(a_{0}\right)\right)+\sum_{h=1}^{k} v\left(P_{\pi}\left(b_{h}\right)\right) .
$$

$$
\begin{aligned}
& \bar{P}_{\pi}\left(a_{0}\right)=\emptyset \text { and } S \cup \bar{P}_{\pi}\left(a_{k}\right)=\bar{P}_{\pi}\left(a_{k}\right) \text { imply } \\
& \qquad \sum_{h=1}^{k} v\left(\bar{P}_{\pi}\left(a_{h}\right)\right) \geq v(S)+\sum_{h=1}^{k} v\left(P_{\pi}\left(b_{h}\right)\right) .
\end{aligned}
$$

Since $\sum_{i \in S_{h}} \bar{m}_{i}^{v}(\pi)=v\left(\bar{P}_{\pi}\left(a_{h}\right)-v\left(P_{\pi}\left(b_{h}\right)\right), h=1, \ldots, k\right.$, and $\sum_{i \in S} \bar{m}_{i}^{v}(\pi)=\sum_{h=1}^{k} \sum_{i \in S_{h}} \bar{m}_{i}^{v}(\pi)$, we obtain $\sum_{i \in S} \bar{m}_{i}^{v}(\pi) \geq v(S)$ 


\section{Axiomatization for cycle digraph games}

On the subclass of cycle-free digraph games the Shapley value for digraph games coincides with the Shapley value for games with precedence constraints of Faigle and Kern [2]. Thus, the axiomatization of the latter value obtained in [2] serves also for the Shapley value for cycle-free digraph games. Now we obtain an axiomatization of the Shapley value on another subclass of $\mathcal{G}_{N}^{\Gamma^{c}}$, the subclass of cycle digraph games. Remark 3.2 implies that a directed cycle on a player set is a connected digraph, every node of which is an undominated player.

Theorem 4.1 The Shapley value for digraph games is the unique value on $\mathcal{G}_{N}^{\Gamma^{c}}$ that meets $E, L, R H M$, and $S S D C$.

Proof. I [Existence]. The proof is similar to that of Proposition 3.2 concerning E, L, $\mathrm{HM}$, and SSDC. For RHM in comparison to HM we only need to add that due to Remark 3.2 all hierarchical coalitions involved are connected.

II [UniQUeness]. First prove that on $\mathcal{G}_{N}^{\Gamma^{c}}$ E, RHM, and SSDC imply RHNP. Take any $(v, \Gamma) \in \mathcal{G}_{N}^{\Gamma^{c}}$ with restricted hierarchical null-player $i$ and let $v_{0}(S)=0$ for all $S \subseteq N$. Hence, $m_{i}^{v}(S)=0=m_{i}^{v_{0}}(S)$ for all $S \subseteq N \backslash\{i\}$ with $S, S \cup\{i\} \in H^{c}(\Gamma)$ and $i \in U^{\Gamma}(S \cup\{i\})$. RHM implies $\xi_{i}(v, \Gamma)=\xi_{i}\left(v_{0}, \Gamma\right)$. E and SSDC imply $\xi_{j}\left(v_{0}, \Gamma\right)=0, j \in N$. Whence, $\xi_{i}(v, \Gamma)=0$.

Since unanimity games form a basis in $\mathcal{G}_{N}$, due to $\mathrm{L}$ it suffices to show that $\xi\left(u_{T}, \Gamma\right)$ is uniquely determined for all $\left(u_{T}, \Gamma\right) \in \mathcal{G}_{N}^{\Gamma^{c}}, T \subseteq N, T \neq \emptyset$.

If $T=N$, then E and SSDC imply $\xi_{i}\left(u_{N}, \Gamma\right)=\frac{1}{n}$ for all $i \in N$.

If $T \in C^{\Gamma}(N), T \neq N$, then due to Remark $3.2 U^{\Gamma}(T)=\{r\}$ for some $r \in T$. For all $i \in$ $T \backslash\{r\}$ and $S \subseteq N \backslash\{i\}$ such that $S, S \cup\{i\} \in H^{c}(\Gamma)$ and $i \in U^{\Gamma}(S \cup\{i\}), m_{i}^{u_{T}}(S)=m_{i}^{u_{N}}(S)$. RHM implies $\xi_{i}\left(u_{T}, \Gamma\right)=\xi_{i}\left(u_{N}, \Gamma\right)=\frac{1}{n}, i \in T \backslash\{r\}$. Since every $i \in N \backslash T$ is a null-player in $u_{T}$, then by Remark 3.3 each $i \in N \backslash T$ is a restricted hierarchical null-player in $\left(u_{T}, \Gamma\right)$ and by RHNP $\xi_{i}\left(u_{T}, \Gamma\right)=0$. E implies $\xi_{r}\left(u_{T}, \Gamma\right)=1-\frac{|T|-1}{n}$.

Finally, take any $T \notin C^{\Gamma}(N)$. Let $T / \Gamma=\left\{T_{1}, \ldots, T_{k}\right\}$, then $U^{\Gamma}\left(T_{h}\right)=\left\{r_{h}\right\}$ for some $r_{h} \in T_{h}, h=1, \ldots, k$. Each $i \in N \backslash T$ is a restricted hierarchical null-player in $\left(u_{T}, \Gamma\right)$ and for all $i \in T \backslash\left\{r_{1}, \ldots, r_{k}\right\}$ and $S \subseteq N \backslash\{i\}$ such that $S, S \cup\{i\} \in H^{c}(\Gamma)$ and $i \in U^{\Gamma}(S \cup\{i\})$, $m_{i}^{u_{T}}(S)=m_{i}^{u_{N}}(S)$. RHNP implies $\xi_{i}\left(u_{T}, \Gamma\right)=0, i \in N \backslash T$, and RHM implies $\xi_{i}\left(u_{T}, \Gamma\right)=\frac{1}{n}$, $i \in T \backslash\left\{r_{1}, \ldots, r_{k}\right\}$. For given $h \in\{1, \ldots, k\}$, let $T^{h} \in C^{\Gamma}(N)$ be the unique smallest connected set containing $T$ such that $U^{\Gamma}\left(T^{h}\right)=\left\{r_{h}\right\}$. Then each $i \in N \backslash T^{h}$ is a restricted hierarchical null player in $\left(u_{T^{h}}, \Gamma\right)$ and for all $i \in T^{h} \backslash\left\{r_{h}\right\}$ and $S \subseteq N \backslash\{i\}$ such that $S, S \cup\{i\} \in H^{c}(\Gamma)$ and $i \in U^{\Gamma}(S \cup\{i\}), m_{i}^{u_{T h}}(S)=m_{i}^{u_{N}}(S)$. RHNP implies $\xi_{i}\left(u_{T^{h}}, \Gamma\right)=0$, $i \in N \backslash T^{h}$, and RHM implies $\xi_{i}\left(u_{T^{h}}, \Gamma\right)=\frac{1}{n}, i \in T^{h} \backslash\left\{r_{h}\right\}$. E implies $\xi_{r_{h}}\left(u_{T^{h}}, \Gamma\right)=1-\frac{\left|T^{h}\right|-1}{n}$. Since for all $S \subseteq N \backslash\left\{r_{h}\right\}$ satisfying $S, S \cup\left\{r_{h}\right\} \in H^{c}(\Gamma)$ and $r_{h} \in U^{\Gamma}\left(S \cup\left\{r_{h}\right\}\right)$ it holds that $m_{r_{h}}^{u_{T}^{h}}(S)=m_{r_{h}}^{u_{T}}(S)$, RHM implies $\xi_{r_{h}}\left(u_{T}, \Gamma\right)=\xi_{r_{h}}\left(u_{T^{h}}, \Gamma\right)=1-\frac{\left|T^{h}\right|-1}{n}$.

Remark 4.1 The classical Shapley value is axiomatized in Young [10] by efficiency, equal treatment property, and marginality, without a priori requirement of additivity. However, for the axiomatization of the Shapley value for digraph games on the subclass of cycle digraph games we need both linearity and restricted marginality. The induction argument of Young does not work in this case because the decomposition of a TU game is considered via the unanimity basis determined by all possible coalitions, but opposite to marginality in Young [10], restricted marginality considers only the hierarchical coalitions, which form here a proper subset of the set of all coalitions.

Acknowledgements The research of the first author was supported by the RFBR-NSFC grant RF-CN 13.01.91160. The research was done during her stay at the University of Twente, whose hospitality is appreciated. 


\section{References}

[1] Aumann, R.J., Drèze, J. (1974). Cooperative games with coalitional structures. International Journal of Game Theory, 3, 217-237.

[2] Faigle, U., Kern, W. (1992). The Shapley value for cooperative games under precedence constraints. International Journal of Game Theory, 21, 249-266.

[3] Gillies, D.B. (1953). Some Theorems on $n$-Person Games, Ph.D. thesis, Princeton University, Princeton.

[4] Gilles, R.P., Owen G. (1991). Games with permission structures: The disjunctive approach. Working Paper E92-04, Virginia Polytechnic Institute and State University, Blacksburg.

[5] Gilles, R.P., Owen G., van den Brink, R. (1992). Games with permission structures: The conjunctive approach. International Journal of Game Theory, 20, 277-293.

[6] Khmelnitskaya, A.B., Selçuk, Ö, Talman A.J.J. (2012). The average covering tree value for directed graph games, CentER Discussion Paper 2012-037, CentER, Tilburg University, Tilburg.

[7] Myerson, R.B. (1977). Graphs and cooperation in games. Mathematics of Operations Research, 2, 225-229.

[8] Owen, G. (1977). Values of games with a priori unions. In: Henn, R., Moeschlin, O. (Eds.), Essays in Mathematical Economics and Game Theory. Springer-Verlag, Berlin, pp. $76-88$.

[9] Shapley, L.S. (1953). A value for n-person games. In: Kuhn, H.W., Tucker, A.W. (Eds.), Contributions to the Theory of Games II. Princeton University Press, Princeton, pp. 307-317.

[10] Young, H.P. (1985). Monotonic solutions of cooperative games. International Journal of Game Theory, 14, 65-72. 